\title{
Evaluation of magnetic field homogeneity using in-out signal cycle mapping in gradient recalled echo images of a mixed water/oil phantom as a rough indication for daily quality control
}

\author{
Yoshiyuki Ishimori * Hiraku Kawamura, Masahiko Monma \\ Department of Radiological Sciences, Ibaraki Prefectural University of Health Sciences, Ibaraki, Japan
}

Received: March 30, 2016

Accepted: June 11, 2016

Online Published: June 24, 2016

DOI: $10.5430 /$ jbgc.v6n2p7

URL: http://dx.doi.org/10.5430/jbgc.v6n2p7

\begin{abstract}
Objective: Magnetic field $\left(\mathrm{B}_{0}\right)$ homogeneity is important for the performance of a magnetic resonance imaging (MRI) system. Traditionally, $\mathrm{B}_{0}$ homogeneity was measured using the spectral peak or phase-mapping methods. However, these procedures are not generally accessible to the MRI operator and are rarely performed routinely. This study proposes a novel method for measuring $\mathrm{B}_{0}$ homogeneity that can be implemented in daily quality control (QC).

Methods: When a uniformly mixed water/oil phantom was imaged using a gradient recalled echo (GRE) pulse sequence, the signal intensity dynamically changed with echo time (TE). From this, the resonant frequency was calculated with a simplex curve-fitting algorithm on a pixel-by-pixel basis. The standard deviation of resonant frequency $\left(\mathrm{SD} \nu_{0}\right)$ was used as the index of $\mathrm{B}_{0}$ homogeneity. The appropriate TE pattern and feasibility of $\mathrm{B}_{0}$ homogeneity evaluation were examined.

Results: Over seven TEs (choosing nominal in-phase, out-phase, and the midpoints of both) were required to measure stable $\mathrm{SD} \nu_{0}$ in a 1.5-T scanner. As $\mathrm{B}_{0}$ homogeneity worsened, the $\mathrm{SD} \nu_{0}$ became larger at the off-center position. Although a positive correlation was observed with the width of the spectral peak obtained by the phase-difference method, the $\mathrm{SD} \nu_{0}$ value was about $5 \times 10^{4}$ times greater. Therefore, $\mathrm{SD} \nu_{0}$ can be used only as an index of $\mathrm{B}_{0}$ homogeneity. Similar results were obtained using a 0.3-T scanner. A $\nu_{0}$ map and SD $\nu_{0}$ can be obtained by acquiring several GRE images of a water/oil mixed phantom within a few minutes.

Conclusions: In-out signal cycle mapping can be easily implemented for daily QC in all MRI scanners.
\end{abstract}

Key Words: Magnetic field $\left(\mathrm{B}_{0}\right)$ homogeneity, Quality control, Chemical shift, In-phase, Out-phase

\section{INTRODUCTION}

Magnetic field $\left(\mathrm{B}_{0}\right)$ homogeneity is an important performance factor of a magnetic resonance imaging (MRI) system. Insufficient $\mathrm{B}_{0}$ homogeneity results in geometrical distortion or nonuniformity of signal intensity in MRI. ${ }^{[1,2]}$ In magnetic resonance spectroscopy (MRS), this causes a broadening of the spectral line width, which results in loss of resolution. ${ }^{[3,4]}$

The MRI quality control (QC) manual published by the American College of Radiology (ACR) describes four methods for measuring $B_{0}$ homogeneity: the spectral peak, bandwidth-

\footnotetext{
*Correspondence: Yoshiyuki Ishimori; Email: ishimori@ipu.ac.jp; Address: Department of Radiological Sciences, Ibaraki Prefectural University of Health Sciences, 4669-2 Ami, Ami-machi, Inashiki-gun, Ibaraki 300-0394, Japan. 
difference, phase-map, and phase-difference methods. ${ }^{[5]}$ However, these procedures are not generally accessible to the MRI system operator and are rarely performed routinely. Conversely, the International Electrotechnical Commission (IEC) standards only describe the measurement of the center frequency and do not specify the measurement of $\mathrm{B}_{0}$ homogeneity. ${ }^{[6]}$ Although some protocols and phantoms have been proposed for the QC of MRI, none correspond to $\mathrm{B}_{0}$ homogeneity measurement. ${ }^{[7-10]}$ Moreover, geometric distortion correction is commonly supplied with most modern MRI systems, but these protocols and phantoms also do not enable monitoring of $\mathrm{B}_{0}$ homogeneity..$^{[1,2]}$ Recently, some MR scanners have been developed with the capability of evaluating $\mathrm{B}_{0}$ homogeneity using the field map generated for water/fat separation; however, this technique is also not common. ${ }^{[11]}$ The present study proposes a novel method for measuring $\mathrm{B}_{0}$ homogeneity that can be implemented in daily QC. The proposed method acquires several images using a simple imaging technique. The pulse sequence required is implemented in all MRI systems.

\section{Methods}

\subsection{In-out signal cycle mapping}

When a uniformly mixed water/oil phantom is imaged using a gradient recalled echo (GRE) pulse sequence, the signal intensity changes dynamically with echo time (TE). This phenomenon is caused by the repetitiveness of the phase change of water and oil, so-called in-phase or out-phase. The behavior of the signal change can be approximated by the following equation:

$$
S(T E)=\left\{a \cdot \sin \left(\omega_{c} \cdot T E+b\right)+c\right\} \cdot \exp \left(-T E / T_{2}^{*}\right)
$$

where $\mathrm{S}(\mathrm{TE})$ is the signal intensity at $\mathrm{TE}, \omega_{c}$ is the frequency of signal change by the chemical shift in $[\mathrm{rad} / \mathrm{s}], \mathrm{T}_{2} *$ is the $\mathrm{T}_{2} *$ value of the subject, and $\mathrm{a}, \mathrm{b}$, and $\mathrm{c}$ are constants. For a given value of $\omega_{c}$, the resonant frequency $\nu_{0}$ can be derived as follows:

$$
\nu_{0}=\left(\omega_{c} / 2 \pi\right)\left(3.5 \times 10^{6}\right)[\mathrm{Hz}]
$$

$\omega_{c}$ can be derived from the signal change in multiple TE GRE images by curve fitting. A $\nu_{0}$ map can be created by converting $\omega_{c}$ pixel by pixel (see Figure 1). A histogram of the pixel values of the $\nu_{0}$ map is equivalent to the spectrum of ${ }^{1} \mathrm{H}$. The $\mathrm{B}_{0}$ homogeneity can be evaluated from the full width at half maximum (FWHM) of the spectrum. In this study, the standard deviation of $\nu_{0}\left(\mathrm{SD} \nu_{0}\right)$ was used as the index of $\mathrm{B}_{0}$ homogeneity instead of FWHM as the aim was to develop a simple method acceptable for daily QC.

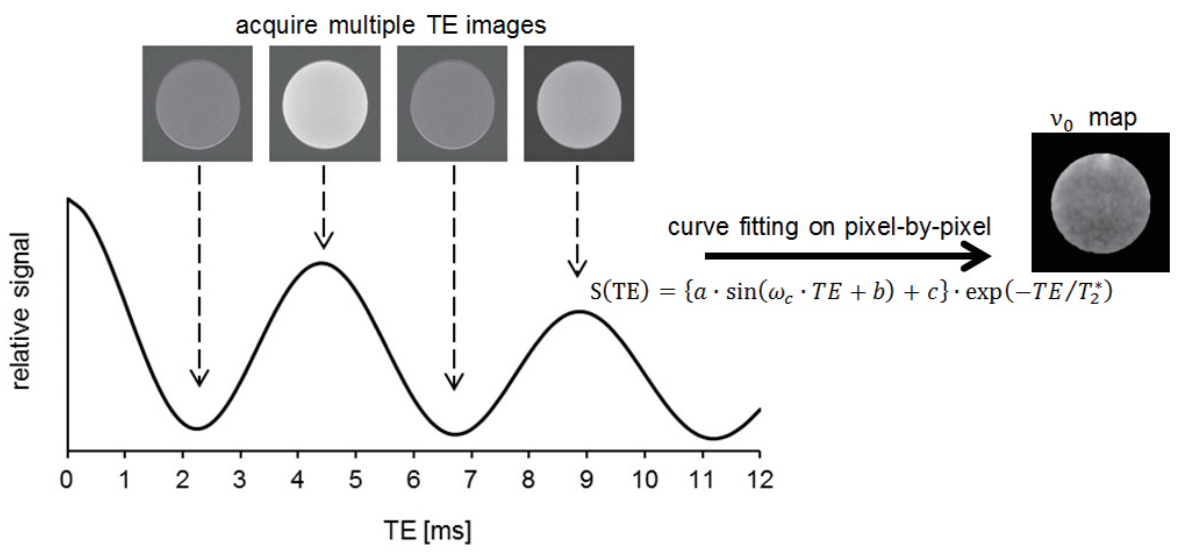

Figure 1. Conceptual diagram of creating a resonant frequency $\left(\nu_{0}\right)$ map.

The resonant frequency can be derived from the periodic signal change of water/oil mixed phantom. This signal change occurs because the signal vector of water and oil repeats in-phase and out-phase. $\nu_{0}$ is derived from conversion of in-out signal cycle $\left(\omega_{c}\right)$ in pixel-by-pixel. TE: echo time.

\subsection{Determination of the appropriate TE pattern}

The total acquisition time must be shortened as much as possible if the procedure is to be implemented in daily QC. The appropriate TE pattern for use in the curve fitting was evaluated. Fifty eight images were acquired at TEs that increased in increments of $0.2 \mathrm{~ms}$ from the minimum to maximum. $\mathrm{The} \mathrm{SD} \nu_{0}$ values were compared when the number of images used in the curve fitting was reduced. The TE patterns compared were as follows: (1) reducing the number of images from the longest TE, (2) increasing the TE in equal increment between the minimum and maximum, (3) choosing nominal in-phase TE, out-phase TE, and the midpoints of both. MRI was performed using a 1.5-T MR scanner (Exelart Vantage, Toshiba Medical Systems, Tokyo, Japan) with a quadrature 
body coil. A 2D spoiled GRE pulse sequence was used (repetition time $[\mathrm{TR}]=100 \mathrm{~ms}, \mathrm{TE}=5.6-17.0 \mathrm{~ms}$, flip angle $[\mathrm{FA}]=45^{\circ}$, number of averages $=1$, field of view [FOV] $=256 \mathrm{~mm}$, slice thickness $=10 \mathrm{~mm}$, matrix size $=128 \times$ 128 , number of slices $=11$ ). A phantom was constructed of an acrylic sphere of $20 \mathrm{~cm}$ diameter filled with mayonnaise (Kewpie mayonnaise, Kewpie Corporation, Tokyo, Japan) and placed at the center of the magnet. The manufacturer's shimming procedure was not applied. Images used in curve fitting were transferred to a personal computer (Elite 8300; Hewlett-Packard, Palo Alto, CA, USA). All analyses were performed on a pixel-by-pixel basis by use of an in-house macro language using ImageJ public domain software (National Institutes of Health, USA). A simplex curve-fitting algorithm is employed in the ImageJ macro function. ${ }^{[12]}$

\subsection{Comparison with the phase-difference method}

To confirm the applicability for $\mathrm{B}_{0}$ homogeneity evaluation, the results obtained by in-out signal cycle mapping were compared with those obtained by the conventional phasedifference method. In the phase-difference method, the phase change $(\Delta \varphi)$ between the different TEs was converted to the resonant frequency $\left(\nu_{0}\right)$ pixel by pixel according to the following equation:

$$
\nu_{0}=(\Delta \varphi / 2 \pi) / 0.0044[\mathrm{~Hz}]
$$

TEs of 9.0 and $13.4 \mathrm{~ms}$ were chosen to place the water and oil in phase, allowing separation of $\mathrm{B}_{0}$ and chemical shift. In in-out signal cycle mapping, the appropriate TE combination obtained from the previous experimental outcome was used. The FWHM values of the histogram of pixel values in the $\nu_{0}$ map were compared between the two methods. The results obtained when the phantom was positioned at the center and when it was off center by $100 \mathrm{~mm}$ in the $\mathrm{z}$-direction were compared. The other imaging conditions and equipment were the same as those for the previous experiment.

\subsection{Adaptability to other equipment}

To verify the adaptability of in-out signal cycle mapping to any other equipment, similar experiments were conducted using a permanent magnet scanner. First, 160 images were acquired at TE increasing by increments of $0.2 \mathrm{~ms}$ from the minimum to maximum. The $\mathrm{SD} \nu_{0}$ values were compared when the number of images used in the curve fitting was reduced. The TE patterns compared were equivalent to those used with the 1.5-T scanner. Then, the $\mathrm{SD} \nu_{0}$ and $\nu_{0}$ maps were compared with respect to the phantom position using the appropriate TE combination. The phantom positions were at the center of the magnet and off center by $80 \mathrm{~mm}$ in the $\mathrm{x}$-direction. A comparison with the phase-difference method was not conducted because the relevant raw data could not be obtained with this scanner. MRI was performed using a 0.3-T MR scanner (AIRIS Vento, Hitachi Medical Corporation, Tokyo, Japan) with a quadrature head coil. A 2D spoiled GRE pulse sequence was used $(\mathrm{TR} / \mathrm{TE}=100 / 3.2$ to $35 \mathrm{~ms}, \mathrm{FA}=60^{\circ}$, number of averages $=1, \mathrm{FOV}=256 \mathrm{~mm}$, slice thickness $=10 \mathrm{~mm}$, matrix size $=128 \times 128$ ).

\section{ReSUlts}

\subsection{Determination of the appropriate TE pattern}

Figure 2 shows the $\mathrm{SD} \nu_{0}$ value measured in the $\nu_{0}$ maps by various TE patterns. Almost the same $\mathrm{SD} \nu_{0}$ values were obtained when using over 30 TEs with the $0.2 \mathrm{~ms}$ increment pattern. Over 16 TEs were required when the even increment pattern was used (i.e., increments less than $0.8 \mathrm{~ms}$ ). Over 7 TEs were required when the nominal feature TEs were used.

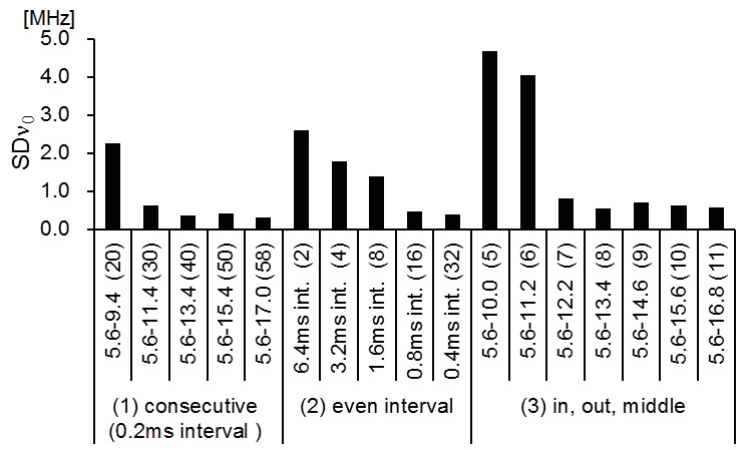

Figure 2. How the standard deviation of the resonant frequency $\left(\mathrm{SD} \nu_{0}\right)$ varies with the different $\mathrm{TE}$ patterns used in the curve fitting.

The TE patterns were (1) $0.2 \mathrm{~ms}$ increment from the minimum TE, (2) even spaced increment between the minimum and maximum, (3) nominal in-phase TE, out-phase TE, and the midpoints of both. The label for each bar gives the TE range or increment used. The number of echoes is shown in parentheses. To obtain a stable measurement value, over 30 TEs were required in case (1), over 16 TEs in case (2), and over 7 TEs in case (3).

\subsection{Comparison with the phase-difference method}

Figure 3 shows plots of pixel values for the $\nu_{0}$ map $\left({ }^{1} \mathrm{H}\right.$ spectrum) according to the different evaluation methods and for different phantom positions. A comparison of the phantom positions showed that the spectral peak became lower and the line width became broader at the off-center position in both methods. The spectral shape obtained by the phasedifference method was asymmetrical. The FWHM values of the spectra were $34.5 \mathrm{~Hz}(0.54 \mathrm{ppm})$ at the center of the magnet and $46.6 \mathrm{~Hz}(0.73 \mathrm{ppm})$ at $100 \mathrm{~mm}$ off center. These values were reasonable according to the specification. Conversely, the spectral shape obtained by in-out signal cycle mapping showed a symmetric shape similar to a Lorentzian 
curve. However, the FWHM of the spectra was about $5 \times$ method.

$10^{4}$ times greater than that obtained by the phase-difference



(a)

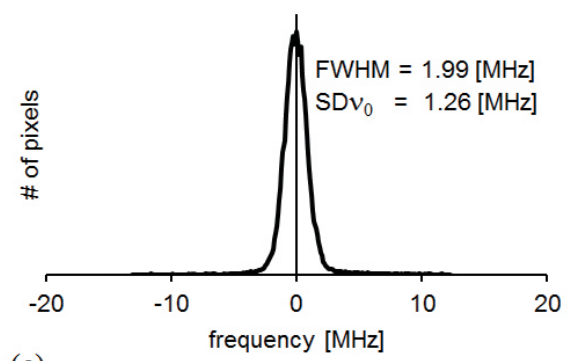

(c)

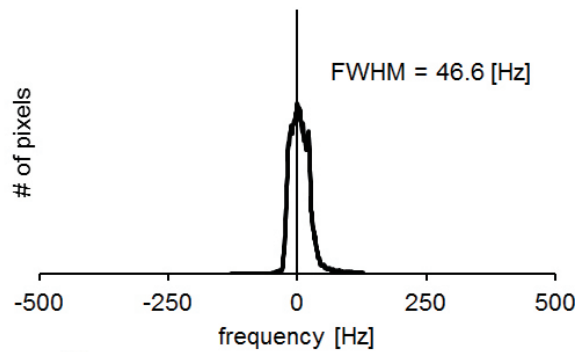

(b)

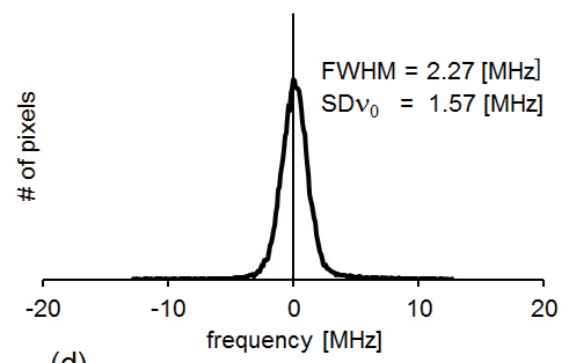

(d)

Figure 3. Plots of pixel values of the $\nu_{0}$ map $\left({ }^{1} \mathrm{H}\right.$ spectrum) using the two evaluation methods at two different phantom positions.

(a) (b) The phase-difference method. (c) (d) In-out signal cycle mapping. (a) (c) At the center of the magnet, (b) (d) $100 \mathrm{~mm}$ off center in the z-direction. The full width at half maximum (FWHM) values are indicated for each spectrum. The standard deviation values of the resonant frequency $\left(S D \nu_{0}\right)$ are also indicated on the results for in-out signal cycle mapping. In the comparison of the phantom position, the spectral peak became lower and the line width became broader at the off-center position in both methods.

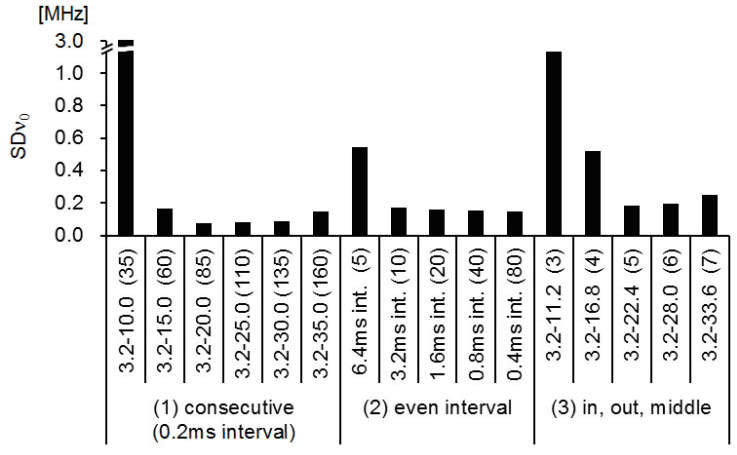

Figure 4. How the standard deviation of the resonant frequency $\left(\mathrm{SD} \nu_{0}\right)$ varied with the TE patterns used in the curve fitting using a $0.3-\mathrm{T}$ scanner.

The TE patterns are equivalent to those in Figure 2. To obtain a stable measurement value, over 60 TEs were required in case (1), over 10 TEs in case (2), and over 5 TEs in case (3).

\subsection{Adaptability to other equipment}

Figure 4 shows how the $\mathrm{SD} \nu_{0}$ value measured in the $\nu_{0}$ maps varied according to various TE patterns using a 0.3-T scanner. Very similar $\mathrm{SD} \nu_{0}$ values were obtained when using 60 or more TEs with the $0.2 \mathrm{~ms}$ increment pattern. Over 10 TEs were required when the even increment pattern used (i.e., increments less than $3.2 \mathrm{~ms}$ ). Over 5 TEs were required when the nominal feature TEs were used.

Figure 5 shows $\nu_{0}$ maps obtained using a 0.3-T scanner when the phantom was positioned at the center of magnet and off center by $80 \mathrm{~mm}$. Inhomogeneous $\mathrm{B}_{0}$ was observed in the $\nu_{0}$ map when the phantom was in the off-center position. $\mathrm{SD} \nu_{0}$ was $0.22 \mathrm{MHz}$ at the center of magnet and $0.29 \mathrm{MHz}$ when off center by $80 \mathrm{~mm}$.



(a)

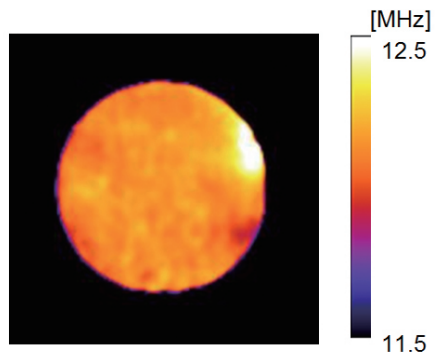

(b)
Figure 5. Resonant frequency $\left(\nu_{0}\right)$ maps using a $0.3-\mathrm{T}$ scanner with the phantom positioned at the center of the magnet (a) and off center by $80 \mathrm{~mm}$ (b).

Inhomogeneous $B_{0}$ was observed at the off-center position. 


\section{Discussion}

In the spectral peak method, the FWHM of the ${ }^{1} \mathrm{H}$ spectrum is measured as an index of $\mathrm{B}_{0}$ homogeneity. However, not all MRI scanners have the capability to acquire MRS, especially those with lower magnetic fields. Even in high magnetic field scanners, it is difficult to set the acquisition volume arbitrarily. Therefore, the spectral peak method is rarely available for routine testing of $\mathrm{B}_{0}$ homogeneity. Moreover, the Lorentzian absorption spectral peak is defined as $F W H M=\frac{1}{\pi \cdot T_{2}^{*}}[\mathrm{~Hz}]{ }^{[13]}$ Thus, $\mathrm{T}_{2}$ may influence the result of $\mathrm{B}_{0}$ homogeneity. The phantom material must be chosen carefully for the spectral peak method. Many manufacturers seem to have adopted the phase-difference method to adjust $\mathrm{B}_{0}$ homogeneity. ${ }^{[14-17]}$ However, the operators cannot realize the condition of $\mathrm{B}_{0}$ homogeneity because the shimming software runs automatically in most settings. Moreover, the phase difference cannot be calculated because not all MRI scanners have the capability to output the phase information of every image for users. Another method to measure $\mathrm{B}_{0}$ homogeneity has been designed for routine testing. ${ }^{[13]}$ This method uses a pair of images acquired with different receive bandwidths. Although this method does not require MRS and phase calculation, it does require a specialized phantom and software. The aim of the proposed novel method is to be useable for everyday checking of $\mathrm{B}_{0}$ homogeneity.

The proposed method utilizes the fact that the signal intensity of a uniformly mixed water/oil phantom changes periodically with changing TE in GRE images. According to the sampling theorem, it is only necessary to acquire two data points per cycle if the behavior of the signal is a simple sine wave. However, in this study, at least three data points are required because the signal change includes $\mathrm{T}_{2} *$ decay. Although acquiring more data points seems better considering the curvefitting precision, the number of images must be reduced as much as possible to allow implementation of the procedure in daily QC. Using the 1.5-T scanner, the measured $\mathrm{SD} \nu_{0}$ becomes stable at less than 0.8 when using over 30 TEs in the $0.2 \mathrm{~ms}$ increment pattern. This TE range corresponds to approximately 1.5 cycles of in-out signal change. To reduce the number of images, the TE increment is increased; therefore, to obtain equivalent measurements, the increment should be $0.8 \mathrm{~ms}$ or less. Sixteen TEs can be acquired with a TE increment of $0.8 \mathrm{~ms}(5.6-17.6 \mathrm{~ms})$. This corresponds to approximately 3 cycles or less of in-out signal change. This TE pattern is an acceptable alternative to the $0.2 \mathrm{~ms}$ increment pattern because it requires fewer images. When the nominal in-phase TE, out-phase TE, and the midpoints of both are chosen, very similar $\mathrm{SD} \nu_{0}$ values are measured with fewer TEs. Stable $\mathrm{SD} \nu_{0}$ values less than 0.8 are measured using over 7 TEs, corresponding to $5.6-12.2 \mathrm{~ms}$ or

Published by Sciedu Press approximately 1.5 cycles of in-out signal change. Signal intensities show a local maximum at the in-phase TE but a local minimum at the out-phase TE. Apparently, TEs should be chosen at the characteristic point of the in-out signal cycle to achieve acceptable precision in the fewest number of TEs. From these results, for an appropriate TE pattern, we choose the nominal feature TEs covering 1.5 cycle of in-out signal change. Seven TEs $(5.6,6.8,7.8,9.0,10.0,11.2$, and 12.4 ms) are assumed to be appropriate for this MR equipment.

Traditionally, $\mathrm{B}_{0}$ homogeneity is defined as the FWHM of a spectral peak. Although various methods have been proposed to evaluate $\mathrm{B}_{0}$ homogeneity, the methods using phase information are considered to be precise and simple. ${ }^{[5,16-19]}$ Therefore, the results obtained by in-out signal cycle mapping are compared with those obtained from the phase-difference method. The FWHM of the spectrum obtained by the phase-map method shows reasonable values according to the specification. In fact, the shimming procedure implemented in the scanner uses the phase-difference method. ${ }^{[14]}$ Obtaining the phase images is allowed to a user in the equipment used in this study. However, simple subtraction between the different TE images cannot eliminate the phase wrap. The phase change caused in the TE increment is calculated by complex division from two sets of real and imaginary data. ${ }^{[20]}$ Although there are other methods to unwrap the phase, these phase calculations cannot be easily performed by a MRI operator. As $\mathrm{B}_{0}$ homogeneity worsens, the spectral peak becomes lower and the line width becomes broader in both methods at the off-center position. This result suggests the possibility that in-out signal cycle mapping can be used for evaluating $\mathrm{B}_{0}$ homogeneity. In the comparison between the two methods with respect to spectral shape, the spectral shape obtained from the phase-difference method is asymmetrical. The phantom container used in this study is made by connecting a pair of acrylic hemispheres. Acrylic is known to have lowest magnetic susceptibility effects, but the phase seems to be affected by the negligible susceptibility induced by the joint of the pieces. ${ }^{[3,13]}$ Conversely, in-out signal cycle mapping seems insensitive to these susceptibilities, as this method uses the magnitude of signal vectors. In addition, in-out signal cycle mapping uses multiple images. In view of this, apparently, in-out signal cycle mapping has a potentially high robustness. However, the FWHM obtained by in-out signal cycle mapping is about $5 \times 10^{4}$ times greater than that obtained by the phase-difference method. $\mathrm{SD} \nu_{0}$ can reflect the $\mathrm{B}_{0}$ homogeneity but it cannot utilize as the true variation of $\nu_{0}$. An approved manner must be used to obtain precise measurements.

In-out signal cycle mapping is ineffective as a tool for daily QC if it cannot adapt to other MR equipment. From the 
results using the 0.3-T permanent magnet scanner, a stable $\mathrm{SD} \nu_{0}$ can be measured with the minimum number of TEs by choosing the nominal feature TEs, similar to the case with the 1.5-T superconducting magnet scanner. The $\mathrm{SD} \nu_{0}$ value obtained by choosing nominal five feature TEs is 0.19 , which is equivalent to the result obtained using 60 TEs with the $0.2 \mathrm{~ms}$ increment pattern. These five TEs $(3.2,5.6,11.2$, 16.8 , and $22.4 \mathrm{~ms}$ ) are assumed to be appropriate for this MR equipment and correspond to approximately one cycle of in-out signal change. The equivalent measurement is obtained with fewer TEs compared with the 1.5-T scanner. The reasons could be as follows: a shorter TE could be used as the minimum TE that resulted in higher signal intensity, and $\mathrm{T}_{2} *$ decay is easily estimated because the period of the TE range of the in-out signal cycle in the 0.3-T scanner is five times that in the 1.5-T scanner. As $\mathrm{B}_{0}$ homogeneity worsens, $\mathrm{SD} \nu_{0}$ becomes larger for the off-center position in the 0.3-T scanner as well. The measured $\mathrm{SD} \nu_{0}$ values are about onefifth of those with the 1.5-T scanner. This ratio corresponds to that of the resonant frequencies. Therefore, $\mathrm{SD} \nu_{0}$ can be utilized as an index of $\mathrm{B}_{0}$ inhomogeneity, similar to the case with the 1.5-T scanner. Another advantage is that local inhomogeneity can be visible because in-out signal cycle mapping can output a $\nu_{0}$ map. For example, acquiring $\nu_{0}$ map only at the magnet center is acceptable as the daily QC. Complete checkup should be done if a worse image like the Figure $5 \mathrm{~b}$ was acquired.

Abrupt changes in system quality may be detected during routine clinical work. More gradual changes may require regular QC testing for detection. Therefore, doing daily QC is preferable to doing weekly or monthly. ACR recommends doing check of $\mathrm{B}_{0}$ homogeneity annually. ${ }^{[5]}$ One of the reasons of this long interval may be complicacy of the procedure. The proposed method is beneficial in simplicity. It should be noted that the phantom material must be chosen carefully to obtain a suitable and a stable signal for QC. In this point, there is no need to match the phantom material with the tissue equivalent materials. The ACR phantom is filled with a solution of nickel chloride and sodium chloride. ${ }^{[5]}$ In this study, the mayonnaise was used as a water/oil mixed material. The composition of the mayonnaise is $70 \%$ of oil ( $80 \%$ of canola oil and $20 \%$ of soybean oil) and $30 \%$ of water or others. ${ }^{[21]}$ It is well known that oil has nine spectral peaks between 0.9 and $5.3 \mathrm{ppm}$ in $\delta \mathrm{m}$ (chemical shift from tetramethylsilane). ${ }^{[22]}$ This wide range of the chemical shift causes a variation in $\nu_{0}$. Although the phase-difference method can derive $\nu_{0}$ directly according to equation (3), ${ }^{[18]}$ in-out signal cycle mapping includes potentially unstable factors. A wide variation in $\nu_{0}$ may be caused by imprecise curve fitting, unstable signal intensities, the effect of the image noise, and so on. Image noise, the variation in the signal intensity of each pixel, causes variation in $\nu_{0}$ because the calculation is done pixelby-pixel basis. The $\mathrm{T}_{2}$ value of the mayonnaise used in this study was about $30 \mathrm{~ms}$. The longer $T_{2}$ value is preferable to obtain a stable and a sufficient signal level. $\mathrm{T}_{1}, \mathrm{~T}_{2}$, and other effective factors need to be controlled in conjunction with the uniformly mixed water/oil phantom. ${ }^{[24]}$ By matching the dielectric constant, the phantom can be used to measure the uniformity and signal-to-noise ratio. The phantom material must be improved for more precise measurement. Also, the fitting algorithm must be improved. Effect of the fitting algorithm to the result is not negligible. ${ }^{[23]}$ These points seemed to be the reason of the discrepancy in the results obtained by the phase difference and the in-out cycle signal mapping. However, precise measurement is beyond the scope of the present study. The aim of the present study was to assess the clinical utility of a new method for daily QC. Therefore, public domain software was used for the data analysis to be utilized by any MR operator. In this study, $\mathrm{SD} \nu_{0}$ is used as an index of $\mathrm{B}_{0}$ homogeneity. The $\mathrm{SD}$ of the pixel values can be easily measured using the same software. $\mathrm{SD} \nu_{0}$ can be utilized just only as an index of $\mathrm{B}_{0}$ homogeneity, and it differs from a true variation of $\nu_{0}$.

Although further improvement is needed, in-out signal cycle mapping has many merits for use in daily QC: it does not need specialized equipment, the GRE pulse sequence is implemented in all MRI systems, acquisition time is only about 2 min, and so on. Furthermore, this technique can be used to compare performance between different MR scanners, even those made by different manufacturers.

\section{Conclusions}

A $\nu_{0}$ map can be obtained by acquiring several GRE images of a mixed water/oil phantom within a few minutes, and $\mathrm{SD} \nu_{0}$ can be used as an index of $\mathrm{B}_{0}$ homogeneity. This technique can be easily implemented in all MRI scanners because it does not need a specialized process such as MRS or phase calculation.

\section{ACKNOWLEDGEMENTS}

The authors thanks to Masahiro Shionoya R.T. for assistance with MR image data acquisition.

\section{CONFLiCTS OF INTEREST Disclosure}

The authors declare that they have no conflicts of interests. 


\section{REFERENCES}

[1] Wang D, Doddrell DM, Cowin G. A novel phantom and method for comprehensive 3-dimensional measurement and correction of geometric distortion in magnetic resonance imaging. Magn Reson Imaging. 2004; 22(4): 529-542. PMid:15120173. http://dx.doi . org/10.1016/j.mri.2004.01.008

[2] Torfeh T, Hammoud R, McGarry M, et al. Development and validation of a novel large field of view phantom and a software module for the quality assurance of geometric distortion in magnetic resonance imaging. Magn Reson Imaging. 2015; 33(7): 939949. PMid:25882440. http://dx.doi.org/10.1016/j.mri.2 015.04 .003

[3] Leach MO, Collins DJ, Keevil S, et al. Quality assessment in in vivo NMR spectroscopy: III. Clinical test objects: design, construction, and solutions. Magn Reson Imaging. 1995; 13(1): 131137. PMid:7898273. http://dx.doi.org/10.1016/0730-725 X (94) 00089-L

[4] Cecil KM. Proton magnetic resonance spectroscopy: technique for the neuroradiologist. Neuroimaging Clin N Am. 2013; 23(3): 381-392. PMid:23928195. http://dx.doi.org/10.1016/j.nic .2012 .10 .003

[5] Committee on quality assurance in magnetic resonance imaging. 2015 Magnetic resonance imaging quality control manual. Reston, VA: American College of Radiology; 2015.

[6] International standard 62464-1. Magnetic resonance equipment for medical imaging-Part 1: Determination of essential image quality parameters. Geneva, Switzerland: International Electrotechnical Commission, 2007.

[7] Price RR, Axel L, Morgan T, et al. Quality assurance methods and phantoms for magnetic resonance imaging: report of AAPM nuclear magnetic resonance Task Group No. 1. Med Phys. 1990: 17(2): 287295. PMid:2333055. http://dx. doi .org/10.1118/1.596566

[8] Lerski RA, de Certaines JD. Performance assessment and quality control in MRI by Eurospin test objects and protocols. Magn Reson Imaging. 1993; 11(6): 817-833. PMid:8371637 http://dx.doi .o rg/10.1016/0730-725X (93) 90199-N

[9] Ihalainen TM, Lönnroth NT, Peltonen JI, et al. MRI quality assurance using the ACR phantom in a multi-unit imaging center. Acta Oncol. 2011; 50(6): 966-972. PMid:21767198. http://dx. doi.org/10. 3109/0284186X. 2011.582515

[10] Sun J, Barnes M, Dowling J, et al. An open source automatic quality assurance (OSAQA) tool for the ACR MRI phantom. Australas Phys Eng Sci Med. 2015; 38(1): 39-46. PMid:25412885. http://dx.doi.org/10.1007/s13246-014-0311-8

[11] Reeder SB, Pineda AR, Wen Z, et al. Iterative decomposition of water and fat with echo asymmetry and least-squares estimation (IDEAL): application with fast spin-echo imaging. Magn Reson Med. 2005; 54(3): 636-644. PMid:16092103. http://dx.doi.org/10.1002 /mrm. 20624
[12] Nelder JA, Mead R. A simplex method for function minimization Comput. J. 1965; 7(4): 308-313. http://dx.doi.org/10.1093 /comjnl/7.4.308

[13] Chen HH, Boykin RD, Clarke GD, et al. Routine testing of magnetic field homogeneity on clinical MRI systems. Med Phys. 2006; 33(11): 4299-4306. PMid:17153408. http://dx.doi.org/10.1118/1.2 359229

[14] Toshiba Vantage MR operator manual. Toshiba Medical Systems Corporation; 2007.

[15] Hitachi Airis Vento operator manual. Hitachi Medical Corporation; 2014.

[16] Schneider E, Glover G. Rapid in vivo proton shimming. Magn Reson Med. 1991; 18(2): 335-347. PMid:2046515. http://dx.doi.org $/ 10.1002 / \mathrm{mrm} .1910180208$

[17] Kanayama S, Kuhara S, Satoh K. In vivo rapid magnetic field measurement and shimming using single scan differential phase mapping. Magn Reson Med. 1996; 36(4): 637-642. PMid:8892219. http://dx.doi.org/10.1002/mrm. 1910360421

[18] Windischberger C, Robinson S, Rauscher A, et al. Robust field map generation using a triple-echo acquisition. J Magn Reson Imaging. 2004; 20(4): 730-734. PMid:15390143. http://dx.doi .org/10. 1002/jmri. 20158

[19] Zhang Y, Li S, Shen J. Automatic high-order shimming using parallel columns mapping (PACMAP). Magn Reson Med. 2009; 62(4): 1073-1079. PMid:19645006. http://dx.doi.org/10.1002/mrm .22077

[20] Ishimori Y, Monma M, Sakurai H, et al. Fat quantification by use of phase change in dual-echo magnetic resonance imaging. Radiol Phys Technol. 2008; 1(1): 89-94. PMid:20821168. http: //dx.doi.org/10.1007/s12194-007-0010-1

[21] Standard tables of food composition in Japan, fifth revised and enlarged edition 2005. Ministry of education, culture, sports, science and technology. 2005 Jan - [cited 2016 May 23]. Japanese. Available from: http://www.mext.go.jp/b_menu/shingi/gij yutu/gijyutu3/toushin/05031802.htm

[22] Bydder M, Girard O, Hamilton G. Mapping the double bonds in triglycerides. Magn Reson Imaging. 2011; 29(8): 10411046. PMid:21868182. http://dx.doi.org/10.1016/j.mri.2 011.07 .004

[23] Mosconi E, Sima DM, Osorio Garcia MI, et al. Different quantification algorithms may lead to different results: a comparison using proton MRS lipid signals. NMR Biomed. 2014; 27(4): 431-443. PMid:24493129. http://dx.doi.org/10.1002/nbm. 3079

[24] Roe JE, Prentice WE, Hornak JP. A multipurpose MRI phantom based on a reverse micelle solution. Magn Reson Med. 1996; 35(1): 136-141. PMid:8771032. http://dx.doi.org/10.1002/mrm. 1 910350118 